\title{
Anterior dislocation of the lens in homocystinuria
}

\author{
A. R. ELKINGTON, S. S. FREEDMAN, BARRIE JAY, \\ AND PETER WRIGHT \\ From Moorfields Eye Hospital, London, Eastbourne Hospital, and \\ King's College Hospital, London
}

Homocystinuria is of particular interest to ophthalmologists, associated as it is with subluxated or dislocated lenses in over go per cent. of cases. It was first described by Carson and Neill (1962) and well over roo cases have since been reported. The subject has recently been reviewed by Gusworth and Dent (1969) and by François (1970).

It is our purpose to discuss the management of anterior dislocation of the lens in five patients with biochemically proven homocystinuria.

\section{Case reports}

Case 1, a boy born in August, 1957, was referred in February, 1968, with bilaterally subluxated lenses and visual acuities of $6 / 18$ in the right eye and 6/12 in the left with aphakic corrections. Homocystinuria had been diagnosed before referral.

In May, 1968, the left lens dislocated into the anterior chamber resulting in acute secondary glaucoma. The lens was extracted under general anaesthesia with anticoagulant cover. There was slight vitreous loss at the time of the operation and in the immediate postoperative period a vitreous haemorrhage was apparent. This took 2 months to clear completely.

In July and September, 1968, the right lens dislocated into the anterior chamber and on each occasion it was replaced behind the iris by dilating the pupil fully and by pressing on the cornea. On one of these occasions it took an hour to replace the lens.

When the patient was last seen in October, $197 \mathrm{I}$, the corrected visual acuity was $6 / 9$ part in each eye. The ocular tensions were normal. There were peripheral pigmentary changes in the right fundus. Ecothiopate iodide $0 \cdot 125$ per cent. was being instilled twice daily into the right eye to prevent a further anterior dislocation of the lens, weaker miotics having proved inadequate.

\section{Family}

This patient has one normal younger brother and one younger sister who is also a known case of homocystinuria. The sister, who was born in 1965 , has bilaterally subluxated lenses.

Case 2, a girl born in June 1954, presented in July, 1960, with headache and vomiting. Before referral a lumbar puncture had been performed when a dilated pupil had been noted in conjunction with an equivocal plantar response. Examination revealed an anteriorly dislocated left lens with acute secondary glaucoma. A left cyclodiathermy was performed, but the eye remained hard and painful and enucleation was necessary in August, i96o.

In 1966 homocystinuria was diagnosed when patients with ectopia lentis were screened for this condition. 
In April, 1967, she again presented with headache and vomiting. The right lens had dislocated into the anterior chamber and the ocular tension was raised. After full dilatation of the pupil the lens was replaced behind the iris. In spite of prophylactic pilocarpine 2 per cent. three times a day, the lens again dislocated into the anterior chamber when the patient's hair was washed one morning before the instillation of the miotic. The lens was again replaced by dilating the pupil.

When she was last seen in January, 1972, the right lens was dislocated posteriorly. Pilocarpine 4 per cent. drops are being given three times a day to prevent a recurrence of the anterior dislocation of the lens. The corrected visual acuity is $6 / 18$ part.

Family

This patient has a normal twin sister. It is possibly relevant that a female cousin died of a subarachnoid haemorrhage while a student at college.

Case 3, a man born in January, 1942, was first seen in $195^{8}$ when being investigated for a pyrexia. He was hypertensive (BP $210 / 140)$, had signs of hepatitis and nephritis, and was psychotic. He had the general appearance of the Marfan syndrome and had bilateral iridodonesis, although the lenses were not obviously subluxated.

Вy 196 I the right lens had dislocated posteriorly and 2 years later the left lens followed suit.

In August, I966, the left lens dislocated into the anterior chamber. The secondary glaucoma was controlled with glycerine and acetazolamide and the lens was reposited by pressing on the cornea with a glass rod after dilating the pupil with phenylephrine io per cent. drops. The patient was then given prophylactic pilocarpine.

In September, 1966, the pilocarpine was stopped in error and the left lens again dislocated into the anterior chamber causing glaucoma. The lens was reposited as before.

In August, 1968, the right lens dislocated anteriorly in spite of prophylactic pilocarpine. It was successfully returned by pressure on the cornea after extensive mydriasis.

Present treatment consists of pilocarpine 4 per cent. drops twice daily to both eyes and, in spite of the eventful past history, the visual acuity is $6 / 9$ in the right eye and $6 / 6$ in the left with aphakic corrections.

Family

This patient had two brothers with subluxated lenses; one had died of unknown causes, and the other, who was dull, of multiple pulmonary emboli. It is probable that they had both suffered from homocystinuria but they died before the disease was described.

Case 4, a boy born in June, 1965, was first seen in 1970 with anterior dislocation of the left lens and secondary glaucoma. Subluxated lenses had been noted 18 months before. The diagnosis of homocystinuria was confirmed biochemically.

In spite of good mydriasis, the lens remained in the anterior chamber and it proved necessary to replace it by pressing on the cornea with a squint hook under general anaesthesia. The pupil was miosed and a peripheral iridectomy was performed.

Despite pilocarpine 4 per cent. drops three times a day the left lens again dislocated anteriorly io days later. Under general anaesthesia the lens was reposited after 20 minutes' firm pressure on the cornea. Prophylactic ecothiopate iodide $0 \cdot 06$ per cent. drops twice daily to both eyes was initiated but 2 days later the left lens again subluxated into the anterior chamber and this time it took 40 minutes to return it. The patient left hospital with a prescription for ecothiopate iodide $0 \cdot 125$ per cent. drops 6-hourly to the left eye and twice daily to the right eye and returned home overseas. We have since heard that further anterior dislocation of the lens has occurred.

Case 5, a girl born in January, 1967, was admitted from abroad on July i 7, 1970, with bilateral anterior dislocation of the lenses and left acute secondary glaucoma. She was backward and was 
known to have had subluxated lenses for which dyflos drops had been prescribed 2 months before admission. At the end of June, 1970, the miotics had been stopped for 5 days so that an ophthalmic examination could be performed, and for the Io days before admission she had complained of a painful left eye and had been vomiting. Homocystinuria was diagnosed on admission to hospital.

The pupils were dilated with phenylephrine to per cent. drops and the right lens was returned behind the iris. Miosis of the right pupil held the lens in place. The left lens remained in the anterior chamber and on July 19, 1970, under general anaesthesia, an unsuccessful attempt was made to replace it behind the iris. It was eventually couched with an iris repositor through a corneo-scleral incision, and a peripheral iridectomy was performed. Miosis was achieved with intracameral acetylcholine

The child made a speedy recovery and prophylactic miotics were prescribed when she was discharged from hospital.

\section{Discussion}

Each of our five patients presented with two problems: anterior dislocation of the lens and homocystinuria.

An anteriorly dislocated lens creates an ophthalmic emergency, usually producing an acute glaucoma as it did in all our cases. The dilemma is whether to extract the lens or to coax it back through the pupil, in the hope that subsequent miosis will prevent recurrent dislocation.

The removal of a dislocated lens has had its protagonists. Clarke (1939) argued that since 53 per cent. of eyes not operated upon became "economically" blind (i.e. with a visual acuity of $20 / 100$ or less), lens extraction was justified. In the ten eyes from which the lens was removed (after a preliminary peripheral iridectomy), vitreous was lost from seven. In spite of the author's claim that there was visual improvement, it must be noted that the follow-up was short. Chace (1945) reported the results of operating upon four cases of congenital bilateral subluxation of the lens, but concluded that the results in all cases were unsatisfactory; incomplete absorption of soft lens matter, glaucoma, and retinal detachment being major complications.

Chandler (1964) deplored the practice of extracting the dislocated lens, in view of the attendant risks. He stressed that since many patients were children, in whom the vitreous was commonly adherent to the lens, vitreous was frequently lost. He emphasized that, provided the follow-up was adequate, retinal detachments occurred quite frequently. In addition, he maintained that most eyes tolerated a lens in the vitreous perfectly well and that, should glaucoma occur, there was usually some other cause for it.

Spaeth and Barber (1965), Thomas, Hollowell, Peters, Coryell, and Lester (1966), and Garston, Gordon, Hart, and Pollitt (1970) reported extracting anteriorly dislocated lenses in patients with homocystinuria; vitreous was invariably lost. Johnston (1968) described the removal of six lenses from patients with homocystinuria; vitreous loss occurred three times and one patient subsequently developed a retinal detachment. Rahman ( 197 I) reported a patient with this condition who developed secondary glaucoma in both eyes because of anterior dislocation of the lenses; when one lens was removed vitreous was lost. When the second eye was opened the lens fell back into the vitreous and was recovered only by using the vectis (apparently no vitreous was lost).

Only in our Case I was a cataract extraction performed (the left lens was removed with slight vitreous loss, and after 3 years the corrected visual acuity is $6 / 9$ part). When the patient's right lens subsequently dislocated twice it was successfully returned through the pupil by pressure on the cornea. In all cases but one (Case 5) it proved possible to push 
the lens back through the fully dilated pupil, although in Case 4 considerable perseverance was required (continuous pressure with a squint hook on the centre of the cornea was necessary, on the first occasion for 20 and on the second for 40 minutes, before the lens returned behind the iris). In the exceptional Case 5, the lens was couched with an iris repositor through a corneo-scleral incision when prolonged pressure on the cornea failed to replace the lens.

Chandler (1964) pointed out the role of pupillary-block in initiating the forward movement of the lens and advocated a peripheral iridectomy to eliminate this mechanism; he maintained that a patent peripheral iridectomy prevented redislocation. This was not so in Case 5 where, in spite of a patent peripheral iridectomy and prophylactic miotics, the lens redislocated into the anterior chamber, twice whilst under our care and once since discharge from hospital (peripheral iridectomies have now been performed on Cases 4 and 5). The same author insisted that miotics did not necessarily prevent re-dislocation (Case 4 supports this opinion), but it seems prudent that patients should use miotics prophylactically. In those instances in which dislocation occurred whilst miotics were being used, Case 2 had her hair washed one morning before the instillation of pilocarpine, Case 3 was taken off pilocarpine in error, and in Case 5 (who was known to have bilateral subluxated lenses and who was using drops of dyflos) the drops were discontinued to allow mydriasis and examination of the fundi.

Homocystinuria should be suspected in all cases of ectopia lentis, particularly if the patient is suspected of having the Marfan syndrome. Schimke, McKusick, Huang, and Pollack (1965) stated that 5 per cent. of cases of ectopia lentis had homocystinuria and Wollensak ( 1967 ), on studying 23 patients who had earlier been diagnosed as suffering from the Marfan syndrome, found that seven had homocystinuria. Only one of the five cases reported here (Case I) was diagnosed as having homocystinuria before referral.

The screening test for homocystinuria has been discussed by Levy, Shih, and MacCready (I97I). The cyanide-nitroprusside test is easy and quick to perform, an important point when dealing with an emergency. Equivocal cases may require sequential two-way paper chromatography for confirmation of the diagnosis; this takes longer.

Spontaneous thromboses in patients with homocystinuria have been described in most major blood vessels (McKusick, Hall, and Char, 197I) and such disasters can be precipitated by general anaesthesia. Johnston (I968) stated that, of ten patients with homocystinuria who had general anaesthetics, five had major thromboembolic episodes postoperatively, of whom two died. It is now generally believed that increased platelet stickiness is the cause of the thromboses (Spaeth, 1972). The most common cause of homocystinuria is cystathionine synthase deficiency (Mudd, I97 r), and in at least some of these patients supplemental pyridoxine will help to restore the blood chemistry towards normal and so perhaps lessen the risk of thrombosis, as also may a low methionine diet. Initially, however, only one of our patients (Case I) was receiving treatment but, in spite of this, no patient appeared to suffer any ill-effects after general anaesthesia (anaesthesia was prolonged beyond an hour in Cases I and 4).

Prophylactic heparin was used by Johnston (1968); hyphaemas developed in two of his five cases and a large haematoma developed in the thigh in one case at the site of the premedication injection. In Case $\mathrm{I}$, the left lens extraction was carried out under heparin cover; an extensive vitreous haemorrhage was seen postoperatively although this eventually cleared. It appears that the use of anticoagulants is likely to precipitate haemorrhage without conferring any obvious benefits. 
In our opinion an anteriorly dislocated lens in a patient with homocystinuria should be managed medically whenever possible because of the risk of vascular thromboses, particularly after surgical procedures. If surgery is inevitable in order to save the eye, pyridoxine should be prescribed and, if there is time in the preoperative period, a low methionine diet should be instituted. The use of anticoagulants is contraindicated. If the lens cannot be replaced behind the iris by medical means, it should be reposited under general anaesthesia and a peripheral iridectomy performed. The surgical technique should allow immediate postoperative mobilization. Only exceptionally in a child or young adult would it appear reasonable to extract the lens. Care must be taken in dilating the pupils of a patient with homocystinuria because of the risk of precipitating anterior dislocation of the lens.

\section{Summary}

Five patients with anterior dislocation of the lens and homocystinuria are presented, and the literature on these subjects is briefly reviewed. The management of this dual problem is discussed, and it is suggested that, whenever possible, the dislocated lens should be reposited without recourse to general anaesthesia.

\section{References}

Garson, N. A. J., and NEILl, D. W. (1962) Arch. Dis. Childh., 37, 505

CHANDLER, P. A. (1964) Arch. Ophthal. (Chicago), 7r, 765

CHAGE, R. R. (1945) Ibid., 34, 425

GLARKE, C. C. (1939) Ibid., 21, 124

CUSWORTH, D. C., and DENT, C. E. (1969) Brit. med. Bull., 25, $4^{2}$

FRANçoIs, J. (1970) In "Perspectives in Ophthalmology", ed. J. E. Winkelman and R. A. Crone, vol. 2, p. 8I. Excerpta Medica, Amsterdam

gARSTON, J. B., GORDON, R. R., haRT, c. T., and POllitt, R. J. (1970) Brit. J. Ophthal., 54, 248

JOHNSTON, s. s. (I968) Ibid., 52, 25 I

LEVY, H. L., SHIH, V. E., and MACCREAdY, R. A. (I97I) In "Inherited Disorders of Sulphur Metabo-

lism", ed. N. A. J. Carson and D. N. Raine, p. 235. Churchill Livingstone, Edinburgh and London

McKUSICK, v. A., HALL, J. G., and CHAR, F. (I97I) Idem, p. 197

MUDD, s. H. (I97I) Idem, p. 204

Rahman, M. (197 I) Brit. F. Ophthal., 55, 338

schimke, R. N., Mckusick, v. A., huANG, T., and pollack, A. D. (1965) J. Amer. med. Ass., 193, 7 I I SPAeth, G. L. and barber, G. W. (1965) Trans. Amer. Acad. Ophthal. Otolaryng., 69, 912 and vacharat, N. (1972) Brit. 7. Ophthal., 56, 205

thomas, R. P., hOllowell, J. G., PETERs, H. J., CORYell, M. E., and lester, R. H. (1966) J. Amer. med. Ass., 198, 560

WOllensak, J. (1967) Ber. dtsch. ophthal. Ges., 68, 404 